\title{
Differential operators for elliptic genera
}

\author{
Matthias R. Gaberdiel and Christoph A. Keller
}

Using the generalization of Zhu's recursion relations to $N=2$ superconformal field theories, we construct modular covariant differential operators for weak Jacobi forms. We show that differential operators of this type characterize the elliptic genera of $N=2$ superconformal minimal models, and sketch how they can be used to constrain extremal $N=2$ superconformal field theories.

\section{Introduction}

It is well known that there is a deep connection between (rational) conformal field theory and the modular group. The origin of this relation lies in the fact that the correlation functions of a conformal field theory on a torus can depend only on its conformal structure, which is parametrized by elements in the quotient space $\mathbb{H}^{+} / \mathrm{SL}(2, \mathbb{Z})$. Formulated as a function of the modular parameter $\tau \in \mathbb{H}^{+}$, the correlation functions must therefore be modular covariant, i.e., covariant under the action of $\mathrm{SL}(2, \mathbb{Z})$.

The full torus amplitude can be expressed in terms of the chiral characters of the conformal field theory; from this point of view, the modular covariance of the correlation functions then comes from the property of these characters to form a vector-valued modular form. The corresponding representation of the modular group, in particular the modular $S$-matrix, encodes important information about the structure of the conformal field theory; for example, it determines the fusion rules via the Verlinde formula [1].

The mathematical argument establishing the modular covariance properties of the characters (under some weak assumptions) was given some time ago by Zhu [2]. A key step in his argument involved the construction of a modular differential equation that annihilates all the characters of a given conformal field theory. (Modular differential equations were first considered from a slightly different point of view in $[3,4]$, see also [5, 6].) This differential equation always comes from a null vector in the vacuum representation $[7,8]$. One can also turn the logic around and construct differential operators starting from essentially arbitrary Virasoro descendants 
of the vacuum. The resulting differential operators are automatically modular covariant; in fact, they are simply the well-known holomorphic modular differential operators that can be obtained by suitable iterations of the operator

$$
\mathcal{D}^{(s)}=q \frac{d}{d q}-\frac{s}{4 \pi^{2}} G_{2}(q),
$$

where $G_{2}(q)$ is the second Eisenstein series.

For $N=2$ superconformal field theories, the natural generalization of the chiral characters are the elliptic genera. These also have good modular properties; in fact, they define vector-valued weak Jacobi forms [9]. (From a mathematical point of view, this was recently established in [10].) For weak Jacobi forms, however, much less is known about modular differential operators. ${ }^{1}$ The purpose of this note is to construct such operators by generalizing the above method to the $N=2$ case. As we shall see, one is naturally led to introduce "twisted" Eisenstein series [13] (see also [14]) in the process. These twisted Eisenstein series serve the same purpose as $G_{2}$ in the bosonic example above; i.e., they cancel the anomalies introduced by the derivatives with respect to $q$ and $y$. Since their transformation properties under the modular group are much more complicated, it is far from obvious how to combine them with derivatives to obtain the modular covariant operators. The approach presented in this note gives a procedure that automatically leads to modular covariant combinations of (twisted) Eisenstein series and derivatives.

Physically, the interest in analysing the modular differential operators comes from the proposed existence of extremal conformal field theories. A conformal field theory cannot just contain the vacuum and its (super-) Virasoro descendants, since the resulting partition function would not be modular invariant. It is thus necessary to have additional primary fields, and modularity gives an upper bound for the weight of the lowest of these new fields. The analysis for the holomorphic $[15,16]$, non-holomorphic [17] and $N=2$ supersymmetric [18] cases always gives a bound linear in $c$, where the coefficient depends on the additional assumptions. Nonetheless, finding a partition function is only a necessary condition for the existence of a conformal field theory, and it is not clear whether these modular functions are

\footnotetext{
${ }^{1}$ After publication of the first version of this paper, we were made aware of $[11,12]$, in which bilinear differential operators are constructed by generalizing the RankinCohen brackets to weak Jacobi forms. It would be interesting to understand how this construction is related to our results. We thank Anatoly Libgober and the referee for pointing out these references to us.
} 
in fact partition functions of consistent conformal field theories. One is thus motivated to look for additional conditions that have to be satisfied. In $[7,8]$ such a constraint was derived: given the characters of a putative conformal field theory, it is always possible to construct by inspection a modular differential equation that annihilates all of them. From the existence of this differential equation, one can then deduce a null vector relation in the vacuum representation, i.e., obtain insights into some other part of the structure of the theory. A necessary prerequisite for this approach is to have some control over the structure of the modular differential operators. The results of this note should therefore allow one to make progress in this direction for $N=2$ theories; this is briefly sketched in Section 4.2.

The remainder of the note is organized as follows. In the following section, we explain the structure of the recursion relations for the elliptic genus amplitudes. In Section 3, we then find a family of differential operators that map weak Jacobi forms of weight zero and an arbitrary index to weak Jacobi forms of higher weight and the same index. In Section 4, we apply these differential operators to the elliptic genera of $N=2$ minimal models and to extremal $N=2$ conformal field theories. There are a number of appendices where some of the more technical calculations are described.

\section{Recursion relations for elliptic genera}

In the following, we want to derive differential equations for the elliptic genus of an $N=2$ superconformal field theory. The differential equations can be obtained using analogous arguments as those used by Zhu [2] in the analysis of the characters. Below, we shall summarize the relevant formulae; the derivations and a summary of our conventions can be found in Appendix A.

Let us consider the functions

$$
\operatorname{Tr}_{\mathcal{R}}^{q, y}(\mathcal{O})=\operatorname{Tr}_{\mathcal{R}}\left(\mathcal{O} q^{L_{0}-\frac{c}{24}} y^{J_{0}}(-1)^{F}\right)
$$

where $\mathcal{R}$ is the (Ramond) representation over which we take the trace, $J_{0}$ is the zero mode of the $U(1)$ current in the $N=2$ superconformal algebra, $F$ is the fermion number operator and $\mathcal{O}$ is an operator from the vertex operator algebra that we insert. The main idea of the analysis is to derive recursion relations for the functions $\operatorname{Tr}_{\mathcal{R}}^{q, y}(\mathcal{O})$. For example, if $a$ is an element of the vertex operator algebra whose $U(1)$ charge is zero, $J_{0} a=0$, then we have 
the original formulae of Zhu for the bosonic case

$$
\begin{aligned}
\operatorname{Tr}_{\mathcal{R}}^{q, y}\left(o\left(a_{\left[-h_{a}\right]} b\right)\right)= & \operatorname{Tr}_{\mathcal{R}}^{q, y}\left(o\left(a_{\left[-h_{a}\right]} \Omega\right) o(b)\right) \\
& +\sum_{k=1}^{\infty} G_{2 k}(q) \operatorname{Tr}_{\mathcal{R}}^{q, y}\left(o\left(a_{\left[2 k-h_{a}\right]} b\right)\right)
\end{aligned}
$$

and

$$
\begin{aligned}
\operatorname{Tr}_{\mathcal{R}}^{q, y}\left(o\left(a_{\left[-h_{a}-n\right]} b\right)\right)= & (-1)^{n} \sum_{2 k \geq n+1}\left(\begin{array}{c}
2 k-1 \\
n
\end{array}\right) \\
& \times G_{2 k}(q) \operatorname{Tr}_{\mathcal{R}}^{q, y}\left(o\left(a_{\left[2 k-h_{a}-n\right]} b\right)\right), \quad n \geq 1,
\end{aligned}
$$

where only ordinary Eisenstein series $G_{2 k}(q)$ appear. The square bracket modes $a_{[-l]}$ as well as $o(a)$ are defined in Appendix A.

A new phenomenon appears if $a$ has $U(1)$ charge $Q \neq 0$, i.e., if $J_{0} a=$ $Q a \neq 0$ : since the derivation of the recursion relations involves cycling $a$ through the trace, we pick up an additional factor $y^{Q}$, which enters the expression. In particular, following the analysis of [13] (see also Appendix A), one finds the recursion relation

$$
\operatorname{Tr}_{\mathcal{R}}^{q, y}\left(o\left(a_{\left[-h_{a}\right]} b\right)\right)=\sum_{k=1}^{\infty} \hat{G}_{k}\left(q, y^{Q}\right) \operatorname{Tr}_{\mathcal{R}}^{q, y}\left(o\left(a_{\left[k-h_{a}\right]} b\right)\right)
$$

where $\hat{G}_{k}(q, y)$ are twisted Eisenstein series, which we will define below. Note that this is only non-vanishing if $b$ has charge $-Q$. Since we have the relation $\left(L_{[-1]} a\right)_{[n]}=-\left(h_{a}+n-1\right) a_{[n-1]}$, we can obtain similar expressions for lower modes

$$
\begin{aligned}
\operatorname{Tr}_{\mathcal{R}}^{q, y}\left(o\left(a_{\left[-h_{a}-n\right]} b\right)\right)= & (-1)^{n} \sum_{k \geq n+1}\left(\begin{array}{c}
k-1 \\
n
\end{array}\right) \\
& \times \hat{G}_{k}\left(q, y^{Q}\right) \operatorname{Tr}_{\mathcal{R}}^{q, y}\left(o\left(a_{\left[k-h_{a}-n\right]} b\right)\right), \quad n \geq 1,
\end{aligned}
$$

which can be shown by induction. For future use we also note that by setting $b=\Omega$, we obtain $\operatorname{Tr}_{\mathcal{R}}^{q, y}\left(o\left(a_{[-n]} \Omega\right)\right)=0$ for $n>h_{a}$ for all operators $a$, charged or uncharged. 
The twisted Eisenstein series $\hat{G}_{k}(q, y)$ that appear in those expressions are defined for $k \geq 1$ by [13] (see also [14])

$$
\begin{aligned}
\hat{G}_{2 k}(q, y) & =2 \zeta(2 k)+\frac{(2 \pi \mathrm{i})^{2 k}}{(2 k-1) !} \sum_{n=1}^{\infty}\left[\frac{n^{2 k-1} q^{n} y^{-1}}{1-q^{n} y^{-1}}+\frac{n^{2 k-1} q^{n} y}{1-q^{n} y}\right], \\
\hat{G}_{2 k+1}(q, y) & =\frac{(2 \pi \mathrm{i})^{2 k+1}}{(2 k) !} \sum_{n=1}^{\infty}\left[\frac{n^{2 k} q^{n} y^{-1}}{1-q^{n} y^{-1}}-\frac{n^{2 k} q^{n} y}{1-q^{n} y}\right] \\
\hat{G}_{1}(q, y) & =(2 \pi \mathrm{i}) \sum_{n=1}^{\infty}\left[\frac{q^{n} y^{-1}}{1-q^{n} y^{-1}}-\frac{q^{n} y}{1-q^{n} y}\right]+\frac{2 \pi \mathrm{i}}{1-y^{-1}}-\pi \mathrm{i} .
\end{aligned}
$$

As usual, we will use the identification $q=\mathrm{e}^{2 \pi \mathrm{i} \tau}$ and $y=\mathrm{e}^{2 \pi \mathrm{i} z}$. It is easy to see from the definitions that $\hat{G}_{k}(\tau,-z)=(-1)^{k} \hat{G}_{k}(\tau, z)$, so that $\hat{G}_{2 k+1}(\tau, 0)=0$. Furthermore, we also recover the usual Eisenstein series for $z=0, \hat{G}_{2 k}(\tau, 0)=G_{2 k}(\tau)$. Similar to the second Eisenstein series $G_{2}$, the generalized Eisenstein series $\hat{G}_{k}$ transform almost as forms of weight $k$, up to anomalies. More precisely, they are invariant under $\tau \mapsto \tau+1, z \mapsto z$, and under $\tau \mapsto-1 / \tau, z \mapsto z / \tau$ they transform as

$$
(2 \pi \mathrm{i})^{-m} \hat{G}_{m}\left(-\frac{1}{\tau}, \frac{z}{\tau}\right)=\sum_{k=1}^{m} \frac{(-1)^{m-k}}{(m-k) !}(2 \pi \mathrm{i})^{-k} \hat{G}_{k}(\tau, z) z^{m-k} \tau^{k}-(-1)^{m} \frac{z^{m}}{m !} .
$$

The transformation of $\hat{G}_{1}$ is particularly simple

$$
\hat{G}_{1}\left(-\frac{1}{\tau}, \frac{z}{\tau}\right)=\tau \hat{G}_{1}(\tau, z)+(2 \pi \mathrm{i}) z .
$$

For the following, it will be convenient to define rescaled Eisenstein series

$$
\mathcal{G}_{2 n}=\frac{1}{(2 \pi \mathrm{i})^{2 n}} G_{2 n} \quad \text { and } \quad \hat{\mathcal{G}}_{n}=\frac{1}{(2 \pi \mathrm{i})^{n}} \hat{G}_{n} .
$$

\section{Modular covariant differential equations}

With these preparations, we can now construct modular covariant differential operators. Let us first review how this worked in the usual bosonic case. It has been known for some time (for a precise statement see, for example, [2]) that the torus one-point functions in the representation $\mathcal{H}_{j}$ of a state $\psi$ with $L_{[0]}$ eigenvalue $h$

$$
\operatorname{Tr}_{\mathcal{H}_{j}}\left(o(\psi) q^{L_{0}-\frac{c}{24}}\right)
$$


transform as a vector-valued modular form of weight $h$. If we take $\psi$ to be of the form $u_{l}=L_{[-2]}^{l} \Omega$, then we can use the recursion relation (2.2) to rewrite $(3.1)$ in terms of $o\left(L_{[-2]} \Omega\right) o\left(u_{l-1}\right)$, as well as one-point functions of states with lower conformal dimension. We can then replace the zero mode $o\left(L_{[-2]} \Omega\right)$ by the differential operator $D_{q}=q \frac{d}{d q}$ because

$$
o\left(L_{[-2]} \Omega\right) q^{L_{0}-\frac{c}{24}}=(2 \pi \mathrm{i})^{2}\left(L_{0}-\frac{c}{24}\right) q^{L_{0}-\frac{c}{24}}=(2 \pi \mathrm{i})^{2} D_{q} q^{L_{0}-c / 24} .
$$

By induction on $l$, we can then write the one-point function (3.1) of $\psi=u_{l}$ in terms of an $l$ th-order differential operator acting on the character (see, for example, [8]). On the other hand, since we know that (3.1) transforms as a modular form of weight $h$, the resulting differential operator must be modular covariant of weight $h$. In fact, this can also be checked explicitly.

For the case of the elliptic genus,

$$
\operatorname{Tr}_{\mathcal{R}}\left(o(\psi)(-1)^{F} y^{J_{0}} q^{L_{0}-\frac{c}{24}}\right)
$$

the situation is more complicated because of the $y^{J_{0}}$ term. In fact, under the modular transformation $\tau \mapsto-1 / \tau, z \mapsto z / \tau$, the one-point function (3.3) of a state $\psi$ with $L_{[0]}$ eigenvalue $h$ can be expressed in terms of the one-point function (3.3) associated to the state $\exp \left(2 \pi \mathrm{i} z J_{[1]}\right) \psi[10]$. The situation is therefore only simple, provided that $\psi$ is annihilated by $J_{[1]}$, for which case (3.3) transforms precisely as a weak Jacobi form of weight $h$ and index $m=\frac{c}{6}$. (For the definition of a weak Jacobi form, see Appendix B.)

Using the recursion relations from Section 2, we can convert the onepoint function of any Neveu-Schwarz $N=2$ descendant of the vacuum into a differential operator (involving now derivatives with respect to $\tau$ and $z$ ) acting on the vacuum amplitude. Starting with a state that is annihilated by $J_{[1]}$, we can thus construct modular covariant differential operators that act on weak Jacobi forms of weight zero. Unfortunately, the analysis is significantly more complicated than in the bosonic case, and we cannot give a closed formula for all $h$. However, we can give explicit formulae for the first few cases, and we can count how many different operators we may obtain in this manner.

\subsection{The operator of weight two}

At $h=1$ the only $N=2$ descendant of the vacuum is the state $J_{[-1]} \Omega$, which is not annihilated by $J_{[1]}$. The first non-trivial vector that is annihilated by 
$J_{[1]}$ appears at $h=2$. In fact, there are two such vectors: $J_{[-2]} \Omega$, which leads to a trivial differential equation, and

$$
\mathcal{M}_{2}=\left(L_{[-2]}-\frac{1}{2 \tilde{c}} J_{[-1]} J_{[-1]}\right) \Omega
$$

where $\tilde{c}=\frac{c}{3}$. Since $\mathcal{M}_{2}$ only involves the $L$ and $J$ modes that have $Q=0$, the old analysis of Zhu applies, and one finds after a short calculation that

$$
\operatorname{Tr}_{\mathcal{R}}^{q, y}\left(o\left(\mathcal{M}_{2}\right)\right)=(2 \pi \mathrm{i})^{2}\left(D_{q}-\frac{1}{4 m} D_{y}^{2}-\frac{1}{2} \mathcal{G}_{2}(q)\right) \operatorname{Tr}_{\mathcal{R}}^{q, y}(\mathbf{1})
$$

where $D_{y}=y \frac{d}{d y}$ and $m=\frac{c}{6}=\frac{\tilde{c}}{2}$. We recognize the differential operator as the well-known (modular covariant) heat kernel operator, whose action on a weak Jacobi form of weight $k$ and index $m$ is defined as

$$
\mathcal{D}_{2}^{(k)}=D_{q}-\frac{1}{4 m} D_{y}^{2}+\frac{2 k-1}{2} \mathcal{G}_{2}(q) .
$$

(In our case, we have $k=0$, since the elliptic genus on the right side of (3.5) has weight zero.)

\subsection{The operator of weight three}

At $h=3$ there are (generically) three states that are annihilated by $J_{[1]}$, namely

$$
\begin{aligned}
& \mathcal{M}_{31}=J_{[-3]} \Omega, \\
& \mathcal{M}_{32}=\left(\tilde{c} L_{[-3]}-J_{[-2]} J_{[-1]}\right) \Omega, \\
& \mathcal{M}_{33}=\left(-\frac{3}{2} \tilde{c}^{2} G_{[-3 / 2]}^{-} G_{[-3 / 2]}^{+}-3 \tilde{c} L_{[-2]} J_{[-1]}+\frac{3}{2} \tilde{c}^{2} L_{[-3]}+J_{[-1]}^{3}\right) \Omega .
\end{aligned}
$$

Both $\mathcal{M}_{31}$ and $\mathcal{M}_{32}$ are $L_{[-1]}$ descendants, and hence give rise to trivial differential operators, since $o\left(L_{[-1]} b\right)=0$ for any state $b$. On the other hand, using the above recursion relations for $\mathcal{M}_{33}$ (that now also involve charged modes), one finds that the associated differential operator is

$$
\begin{aligned}
\mathcal{D}_{3}= & D_{y}^{3}+3 \tilde{c} \mathcal{G}_{2}(q) D_{y}-3 \tilde{c}\left(D_{q}+\mathcal{G}_{2}(q)\right) D_{y}+3 \tilde{c}^{2}\left(\hat{\mathcal{G}}_{1}(q, y) D_{q}\right. \\
& \left.+\hat{\mathcal{G}}_{2}(q, y) D_{y}+\tilde{c} \hat{\mathcal{G}}_{3}(q, y)\right) .
\end{aligned}
$$

Using the explicit modular transformation properties (2.7) and (2.8) of the generalized Eisenstein series, one can check that this differential operator 
really maps a weak Jacobi form of weight zero and index $m=\frac{\tilde{c}}{2}$ to one of weight three and index $m$. One can also show that $\mathcal{D}_{3}$ annihilates the weak Jacobi form $\Phi_{0,1}$, as must be the case since there are no weak Jacobi forms of weight/index $(3,1)$.

\subsection{The operator of weight four}

The analysis at $h=4$ is similar. The space of states that are annihilated by $J_{[1]}$ is seven-dimensional, but the three vectors that are $L_{[-1]}$ descendants of $\mathcal{M}_{31}, \mathcal{M}_{32}$ and $\mathcal{M}_{33}$, all lead to trivial differential operators. The same is true for the state

$$
\mathcal{M}_{41}=\left(J_{[-2]} J_{[-1]}^{2}-2 \tilde{c} J_{[-2]} L_{[-2]}\right) \Omega .
$$

Of the remaining states, $\mathcal{M}_{42}=J_{[-2]}^{2} \Omega$ simply leads to multiplication by $-6 \tilde{c} G_{4}(q)$. The state

$$
\mathcal{M}_{43}=\left(L_{[-2]}^{2}-\frac{1}{\tilde{c}} L_{[-2]} J_{[-1]}^{2}+L_{[-4]}+\frac{1}{4 \tilde{c}^{2}} J_{[-1]}^{4}\right) \Omega
$$

gives $\mathcal{D}_{2}^{(2)} \cdot \mathcal{D}_{2}^{(0)}+\left(\frac{3}{2} \tilde{c}-\frac{7}{2}\right) \mathcal{G}_{4}(q)$, which is the square of the heat kernel plus a $\mathcal{G}_{4}$ term. Finally, the state

$$
\begin{aligned}
\mathcal{M}_{44}= & {\left[(\tilde{c}-2)\left(G_{[-5 / 2]}^{+} G_{[-3 / 2]}^{-}+G_{[-5 / 2]}^{-} G_{[-3 / 2]}^{+}\right)+2 G_{[-3 / 2]}^{-} G_{[-3 / 2]}^{+} J_{[-1]}\right.} \\
& \left.+2 L_{[-2]}^{2}-2 L_{[-3]} J_{[-1]}+2 L_{[-4]}\right] \Omega
\end{aligned}
$$

leads to the differential operator

$$
\begin{aligned}
\mathcal{D}_{4}= & 2\left(D_{q}+2 \mathcal{G}_{2}(q)\right) D_{q}+3 \tilde{c} \mathcal{G}_{4}(q) \\
& +4 \hat{\mathcal{G}}_{1}(q, y)\left(\hat{\mathcal{G}}_{1}(q, y) D_{q}+\hat{\mathcal{G}}_{2}(q, y) D_{y}+\tilde{c} \hat{\mathcal{G}}_{3}(q, y)-\left(D_{q}+\mathcal{G}_{2}(q)\right) D_{y}\right) \\
& -4 \hat{\mathcal{G}}_{2}(q, y)\left((\tilde{c}-2) D_{q}+D_{y}^{2}+\tilde{c} \mathcal{G}_{2}(q)\right)-12(\tilde{c}-1) \hat{\mathcal{G}}_{3}(q, y) D_{y} \\
& -4 \tilde{c}(3 \tilde{c}-4) \hat{\mathcal{G}}_{4}(q, y) .
\end{aligned}
$$

\subsection{The general analysis}

Let us denote the Neveu-Schwarz $N=2$ vacuum representation by $\mathcal{H}$. It is not difficult to determine the number of vectors in $\mathcal{H}$ at fixed conformal 
weight and $U(1)$ charge that are generically annihilated by $J_{[1]}$; they are given by the generating function

$$
Z=\frac{\prod_{r=3 / 2,5 / 2,\left(\left(1+q^{r} y\right)\left(1+q^{r} y^{-1}\right)\right)}^{\infty}}{\prod_{n=2}^{\infty}\left(1-q^{n}\right)^{2}}
$$

To obtain a non-vanishing operator, we need to take a state with vanishing charge. We are thus interested in the coefficients of $y^{0}$, which are given by

$$
Z\left[y^{0}\right]=1+2 q^{2}+3 q^{3}+7 q^{4}+11 q^{5}+23 q^{6}+36 q^{7}+67 q^{8}+\cdots .
$$

We have seen that not all states of a given conformal weight $h$ lead to nontrivial differential operators. Some vectors, for example $J_{[-h]} \Omega$, will vanish identically, while others will lead to Eisenstein series multiplying differential operators of lower degree. However, it is easy to see that the highest derivatives that appear at that conformal weight are of the form:

$$
D_{q}^{l} D_{y}^{h-2 l}, \quad l=0,1, \ldots,\left\lfloor\frac{h}{2}\right\rfloor .
$$

The vectors that give rise to these differential operators (plus correction terms involving lower order derivatives) are the "leading vectors"

$$
v_{l}^{(h)}=L_{[-2]}^{l} J_{[-1]}^{h-2 l} \Omega, \quad l=0,1, \ldots,\left\lfloor\frac{h}{2}\right\rfloor .
$$

They form a spanning set for the quotient space $C_{2}^{\mathrm{NS}}=\mathcal{H} / O_{[2]}^{\mathrm{NS}}(\mathcal{H})$, where $O_{[2]}^{\mathrm{NS}}(\mathcal{H})$ is generated by the vectors ${ }^{2}$

$$
O_{[2]}^{\mathrm{NS}}(\mathcal{H})=\operatorname{span}\left\{L_{[-3-n]} \psi, J_{[-2-n]} \psi, G_{[-3 / 2-n]}^{ \pm} \psi, n \geq 0\right\} .
$$

Now, we want to show that for every conformal weight $h$ and modulo $O_{[2]}^{\mathrm{NS}}(\mathcal{H})$, the space of vectors that are annihilated by $J_{[1]}$ has dimension $\left\lfloor\frac{h}{2}\right\rfloor$. This will imply that for every $h$, there are $\left\lfloor\frac{h}{2}\right\rfloor$ different modular covariant differential operators that differ by their leading terms (3.15).

In fact, it is easy to see that the dimension of this space cannot be bigger than $\left\lfloor\frac{h}{2}\right\rfloor$, since the dimension of $C_{2}^{\mathrm{NS}}$ at conformal weight $h$ is $\left\lfloor\frac{h}{2}\right\rfloor+1$, and

\footnotetext{
${ }^{2}$ The space $O_{[2]}^{\mathrm{NS}}(\mathcal{H})$ does not quite agree with the usual $O_{[2]}(\mathcal{H})$ space, since it contains also the states generated by $G_{[-3 / 2]}^{ \pm}$. Thus, the resulting quotient space $C_{2}^{\mathrm{NS}}$ is also not quite the standard $C_{2}$ space. However, it gives a natural upper bound for the NS-sector version of Zhu's algebra.
} 
since the condition that $J_{[1]}$ annihilates the vector implies that the term $J_{[-1]}^{h} \Omega$ always appears in the combination

$$
\mathcal{M}_{h}=\left(L_{[-2]} J_{[-1]}^{h-2}-\frac{1}{h \tilde{c}} J_{[-1]}^{h}\right) \Omega+\text { other terms. }
$$

To see this, we choose the Poincaré-Birkhoff-Witt basis for the Verma module, where we order the modes so that they appear in the order $\left(L, J, G^{+}\right.$, $\left.G^{-}\right)$. By a direct computation, one finds

$$
J_{[1]} J_{[-1]}^{h} \Omega=h \tilde{c} J_{[-1]}^{h-1} \Omega .
$$

Thus, if $\mathcal{M}_{h}=J_{[-1]}^{h} \Omega+\psi$ is a vector in the Verma of conformal weight $h$ that is annihilated by $J_{[1]}$, then $J_{[1]} \psi=-h \tilde{c} J_{[-1]}^{h-1} \Omega$. Without loss of generality, we may assume that $\psi$ does not involve a term proportional to $J_{[-1]}^{h} \Omega$. Given that $\psi$ has conformal dimension $h$ and $U(1)$ charge zero, it is then of the form

$$
\begin{aligned}
\psi= & a L_{[-2]} J_{[-1]}^{h-2} \Omega+b J_{[-1]}^{h-3} G_{[-3 / 2]}^{+} G_{[-3 / 2]}^{-} \Omega \\
& + \text { terms with less than }(h-1) \text { modes. }
\end{aligned}
$$

Each time we apply a commutator, the number of modes is reduced by at least one. Thus, it follows that

$$
\begin{aligned}
J_{[1]} \psi= & a J_{[-1]}^{h-1} \Omega+a(h-2) \tilde{c} L_{[-2]} J_{[-1]}^{h-3} \Omega+b(h-3) \tilde{c} J_{[-1]}^{h-4} G_{[-3 / 2]}^{+} G_{[-3 / 2]}^{-} \Omega \\
& +b J_{[-1]}^{h-3}\left(2 L_{[-2]}+J_{[-2]}\right) \Omega+\text { terms with less than }(h-2) \text { modes } \\
(3.21)= & a J_{[-1]}^{h-1} \Omega+\text { terms with less than }(h-1) \text { modes. }
\end{aligned}
$$

In order for $\mathcal{M}_{h}=J_{[-1]}^{h} \Omega+\psi$ to be annihilated by $J_{[1]}$, it therefore follows that $a=-h \tilde{c}$. This demonstrates (3.18).

In order to prove that the space is at least as big as claimed, we note from our previous explicit calculations that at conformal weights $h=2,3,4$ there are vectors that are annihilated by $J_{[1]}$, and whose leading terms are

$$
\begin{aligned}
& \mathcal{M}_{2}=\left(L_{[-2]}-\frac{1}{2 \tilde{c}} J_{[-1]}^{2}\right) \Omega, \\
& \mathcal{M}_{3}=\left(L_{[-2]} J_{[-1]}-\frac{1}{3 \tilde{c}} J_{[-1]}^{3}\right) \Omega+O_{[2]}^{\mathrm{NS}}, \\
& \mathcal{M}_{4}=L_{[-2]}^{2} \Omega+O_{[2]}^{\mathrm{NS}} .
\end{aligned}
$$


One also finds that at $h=5$, there is a vector $\mathcal{M}_{5}$ that is annihilated by $J_{[1]}$, and that is of the form

$$
\mathcal{M}_{5}=\left(L_{[-2]} J_{[-1]}^{3}-\frac{1}{5 \tilde{c}} J_{[-1]}^{5}\right) \Omega+O_{[2]}^{\mathrm{NS}}
$$

It is easy to see (by the same arguments as for the usual $C_{2}$ space) that the $C_{2}^{\mathrm{NS}}$ quotient space is a commutative algebra, where the product is defined by $V_{[-h]}(\psi) \chi$, with $h$ the conformal weight of $\psi$. Furthermore, if $\psi$ and $\chi$ are annihilated by $J_{[1]}$, so is their product $V_{[-h]}(\psi) \chi$. Thus, we can generate states that are annihilated by $J_{[1]}$ by taking successive products of low-lying states. It only remains to check that the states we generate are sufficient in number to prove the above claim. Using the commutative algebra structure on $C_{2}^{\mathrm{NS}}$, this is then effectively a problem in a polynomial algebra. Let us define the generators of $C_{2}^{\mathrm{NS}}$

$$
x=L_{[-2]} \Omega, \quad y=\sqrt{\frac{1}{2 \tilde{c}}} J_{[-1]} \Omega,
$$

where $y$ has weight one and $x$ has weight two. Then $\mathcal{M}_{2}, \mathcal{M}_{3}, \mathcal{M}_{4}$ and $\mathcal{M}_{5}$ correspond to the generators

$$
p_{2}=\left(x-y^{2}\right), \quad p_{3}=y\left(x-\frac{2}{3} y^{2}\right), \quad p_{4}=x^{2}, \quad p_{5}=y^{3}\left(x-\frac{2}{5} y^{2}\right),
$$

respectively. It is easy to see that $p_{2}^{3}, p_{2} p_{4}$ and $p_{3}^{2}$ generate at $h=6$

$$
p_{6}=y^{4}\left(x-\frac{1}{3} y^{2}\right) \text {. }
$$

Let us first consider the case when $h$ is even, $h=2 n$, and use induction on $n$. For $h=2$ the statement is obviously true. Suppose $f_{1}, \ldots, f_{n}$ are $n$ linearly independent polynomials of weight $2 n$. At weight $h=2 n+2$, we then consider the polynomials

$$
\begin{aligned}
& p_{2} \cdot f_{j}, \quad j=1, \ldots, n, \quad \text { and } \quad p_{4}^{\frac{n+1}{2}} \quad \text { if } n \text { is odd, } \\
& p_{2} \cdot f_{j}, \quad j=1, \ldots, n, \quad \text { and } \quad p_{6} \cdot p_{4}^{\frac{n-2}{2}} \quad \text { if } n \text { is even. }
\end{aligned}
$$

Obviously, the polynomials $p_{2} \cdot f_{j}$ are linearly independent in each case. By setting $x=y^{2}$, they all vanish, but neither does $p_{4}$ nor $p_{6}$. Thus, the last element in each case is linearly independent of the first $n$ elements, and we have therefore found $\frac{h}{2}=n+1$ linearly independent polynomials. 
For odd $h$ the argument is essentially the same. In constructing the polynomials at $h=2 n+1$ from those at $2 n-1$, we add either $p_{3} \cdot p_{4}^{m}$ with $m=\frac{n-1}{2}$ (if $n$ is odd) or $p_{5} \cdot p_{4}^{m}$ with $m=\frac{n-2}{2}$ (if $n$ is even). This completes the proof.

\section{Applications}

Finally, let us discuss some applications of these general considerations.

\subsection{Modular differential equations of minimal models}

For the case $N=2$ minimal models, one expects that differential operators of the kind discussed above should be the building blocks for the modular differential equation that characterizes all elliptic genera. Recall that the central charge of the $k$ th minimal model is given by

$$
c=\frac{3 k}{k+2}
$$

The elliptic genera of these models were determined in $[9,19]$. Let us first consider the case $k=1$ for which $c=1$, so that $\tilde{c}=\frac{1}{3}$ and $m=\frac{1}{6}$. In this case, the vector $\mathcal{N}=\mathcal{M}_{2}$ given in (3.4) is actually a null vector. This means that the corresponding differential operator must annihilate the elliptic genera

$$
\left(D_{q}-\frac{3}{2} D_{y}^{2}-\frac{1}{2} \mathcal{G}_{2}(q)\right) \chi_{\mathcal{R}}(q, y)=0
$$

A second null vector of the theory can be obtained by applying $G_{[-1 / 2]}^{-} G_{[-1 / 2]}^{+}$ to $\mathcal{N}$, leading to

$$
\hat{\mathcal{N}}=\left(3 G_{[-3 / 2]}^{-} G_{[-3 / 2]}^{+}-2 L_{[-3]}+6 L_{[-2]} J_{[-1]}-3 J_{[-2]} J_{[-1]}-4 J_{[-3]}\right) \Omega .
$$

Note that this is a linear combination of $\mathcal{M}_{31}, \mathcal{M}_{32}$ and $\mathcal{M}_{33}$, as well as $J_{[-1]} \mathcal{N}$. It leads to the differential equation

$$
\left(\left(D_{q}+\mathcal{G}_{2}(q)\right) D_{y}-\hat{\mathcal{G}}_{1}(q, y) D_{q}-\hat{\mathcal{G}}_{2}(q, y) D_{y}-\frac{1}{3} \hat{\mathcal{G}}_{3}(q, y)\right) \chi_{\mathcal{R}}(q, y)=0
$$


The $k=1$ minimal model has three elliptic genera,

$$
\chi_{1}^{0}(q, y)=y^{-1 / 6}+\left(y^{-1 / 6}-y^{5 / 6}\right) q+\left(-y^{-7 / 6}+2 y^{-1 / 6}-y^{5 / 6}\right) q^{2}+\cdots,
$$

$$
\begin{aligned}
\chi_{-1}^{0}(q, y) & =-y^{1 / 6}+\left(-y^{1 / 6}+y^{-5 / 6}\right) q+\left(y^{7 / 6}-2 y^{1 / 6}+y^{-5 / 6}\right) q^{2}+\cdots, \\
\chi_{3}^{0}(q, y) & =\left(-y^{-1 / 2}+y^{1 / 2}\right) q^{1 / 3}+\left(-y^{-1 / 2}+y^{1 / 2}\right) q^{4 / 3}+\cdots .
\end{aligned}
$$

It is straightforward to check that these are indeed annihilated by the two differential equations.

Conversely, one can also show that (4.5) are the only solutions of (4.2) and (4.4). Since all differential operators only contain integer powers of $y$ and $q$, by linearity we can assume without loss of generality that a solution $\left.\chi_{(} q, y\right)$ contains only terms of the form $y^{Q+\mathbb{Z}} q^{h+\mathbb{N}_{0}}$, where $h$ is the lowest power of $q$ that appears and $Q$ is the lowest power of $y$ in a term with $q^{h}$. The power series ansatz for $\chi(q, y)$ is thus

$$
\chi(q, y)=q^{h} y^{Q} \sum_{\ell=0}^{N} c(\ell) y^{\ell}+\mathcal{O}\left(q^{h+1}\right), \quad c(0) \neq 0 .
$$

Since the differential operators never decrease the power of $q$, the terms we have written out explicitly in (4.6) must be annihilated by the differential operators up to terms of order $\mathcal{O}\left(q^{h+1}\right)$. Acting with (4.2), we thus obtain

$$
h=\frac{3}{2} Q^{2}-\frac{1}{24},
$$

while (4.4) leads to

$$
h(1+2 Q)=0
$$

Note that there is a slight subtlety in deriving the second equation because $\hat{\mathcal{G}_{1}}$ contains the term $(1-y)^{-1}$. However, since the elliptic genus is an index, we know that $\chi(q, y)$ must vanish for $y=1$ unless $h=0$. This means that either $D_{q}$ vanishes or that we can factor out $(1-y)$, so that the action of $\hat{\mathcal{G}}_{1}$ is indeed well defined.

The two equations (4.7) and (4.8) must be satisfied simultaneously, and their only solutions are $\left(h=0, Q= \pm \frac{1}{6}\right)$ and $\left(h=\frac{1}{3}, Q=-\frac{1}{2}\right)$. This shows that there are at most three solutions of the form (4.6), and thus, given the existence of the explicit solutions $\chi_{ \pm 1}^{0}$ and $\chi_{3}^{0}$, precisely three such solutions.

The analysis for a general minimal $N=2$ model is similar. The $k$ th minimal model has an uncharged null vector $\mathcal{N}_{k}$ at level $k+1$, and the 
non-trivial descendant $G_{[-1 / 2]}^{-} G_{[-1 / 2]}^{+} \mathcal{N}_{k}$ at level $k+2$. Acting on the lowest powers of $q$ these will again give two polynomial equations for $h$ and $Q$, whose leading terms have weight $k+1$ and $k+2$, respectively. (Here we associate weight one to $Q$ and weight two to $h$.) To find the number of joint solutions, first substitute $h=H^{2}$, so that $H$ and $Q$ have both weight one. In addition, we introduce an auxiliary variable $z$ of weight one and multiply the different terms so as to obtain homogeneous polynomials of degree $k+1$ and $k+2$, respectively. These equations can now be thought of as equations in projective space $\mathbb{P}^{2}$, and Bézout's theorem then implies that there are $(k+1)(k+2)$ solutions, taking into account multiplicities. ${ }^{3}$ Since they obviously come in pairs of $(Q, \pm H)$, it follows that there are at most $(k+1)(k+2) / 2$ solutions for $(Q, h)$. This is exactly the number of independent elliptic genera of the $k$ th minimal model.

\subsection{Modular constraints for extremal self-dual theories}

Modular differential equations gave an interesting constraint for extremal (bosonic) conformal field theories [7,8]. Given our insight into the structure of differential operators for weak Jacobi forms, we can now apply similar techniques to the $N=2$ case. To start with we recall that the space of weak Jacobi forms of even weight $w$ and index $m, \tilde{J}_{m, w}$, is spanned by monomials of the form (see Appendix B)

$$
\left(\tilde{\phi}_{-2,1}\right)^{a}\left(\tilde{\phi}_{0,1}\right)^{b} G_{4}^{c} G_{6}^{d},
$$

where $a+b=m$ and $4 c+6 d-2 a=w$. The number of solutions to the second equation is the number of modular forms of weight $w^{\prime}=w+2 a$, which is proportional to

$$
N\left(w^{\prime}\right)=\frac{w^{\prime}}{12}+\text { subleading. }
$$

Thus the total dimension is, for large $m$, of the form

$$
\operatorname{dim} \tilde{J}_{m, w}=\frac{m^{2}}{12}+\frac{w m}{12}+\text { subleading. }
$$

\footnotetext{
${ }^{3}$ We are assuming here that the two polynomials do not contain a common factor. This should follow from the fact that Zhu's algebra is finite-dimensional for the minimal models, compare the discussion in [20].
} 
Suppose now that we are given a self-dual $N=2$ superconformal field theory at $c=6 \mathrm{~m}$. Its elliptic genus defines a weak Jacobi form of weight zero and index $m$. We want to construct a modular differential equation of weight $w$ that annihilates it. As we have seen, there are $\left\lfloor\frac{w^{\prime}}{2}\right\rfloor$ differential operators of order $w^{\prime}$. Dressing them with an ordinary modular form of weight $w-w^{\prime}$, the total number of differential operators of degree that we can construct in this manner is, for large $w$,

$$
\frac{1}{2} \sum_{w^{\prime}=0}^{w} \frac{w^{\prime}}{2} \frac{w-w^{\prime}}{12}=\frac{w^{3}}{288}+\text { subleading }
$$

where the factor $\frac{1}{2}$ corrects for the fact that there are no modular forms of odd weight. If we apply any such differential operator to the elliptic genus of our given $N=2$ superconformal field theory at $c=6 \mathrm{~m}$, we obtain a weak Jacobi form of weight $w$ and index $m$. Since there are at most $\operatorname{dim}\left(\tilde{J}_{m, w}\right)$ linearly independent weak Jacobi forms of this weight and index, we can always choose a suitable linear combination of the differential operators to annihilate the elliptic genus if

$$
\frac{w^{3}}{288} \geq \frac{m^{2}}{12}+\frac{w m}{12}
$$

i.e., if $w \geq w_{*}$ with

$$
w_{*}=24^{\frac{1}{3}} m^{\frac{2}{3}}=\left(\frac{2}{3}\right)^{\frac{1}{3}} c^{\frac{2}{3}} .
$$

Following the logic of the arguments of $[7,8]$, this would then suggest that the self-dual $N=2$ superconformal field theory has a null vector at conformal weight $w_{*}$. Since the superconformal descendants of the vacuum do not have any null vectors for $c>3$, this would thus imply that the theory has to have additional primary fields at conformal weight $h \sim w_{*}$.

Note that we could also ignore the $N=2$ supersymmetry of the problem and treat the theory from a bosonic point of view. With respect to the bosonic generators, the theory is not self-dual, and the original counting argument needs to be modified slightly; however, this will still lead to a differential equation of weight $w_{*} \sim \sqrt{2 c}$, which is stronger than (4.14). On the other hand, one may hope that the more refined $N=2$ analysis from above will help us to fill the gap in the argument of [8], and thus prove a no-go theorem in this case. 


\section{Acknowledgments}

We thank Sergei Gukov for collaboration at an early stage of this project. We are also very grateful to Claude Eicher, Terry Gannon, Sebastian Gerigk, Roberto Volpato and Don Zagier for helpful discussions. The research of M.R.G. is supported by the Swiss National Science Foundation and C.A.K. is supported by a Fellowship of the Swiss National Science Foundation.

\section{Appendix A. Conventions and recursion relations}

\section{A.1. Conventions}

Let us begin by collecting our conventions. The vertex operator of a state $a$ is given by

$$
V(a, w)=\sum_{n} a_{n} w^{-n-h_{a}} .
$$

Note that we use a different convention than [2] - in particular, $a_{n}=a(h+$ $n-1)$. It is sometimes convenient to introduce a special symbol for the zero mode as

$$
o(a)=a_{0}
$$

The modes that are appropriate for the analysis on the torus are defined by (see Section 4.2 of [2])

$$
V[a, z]=\mathrm{e}^{2 \pi \mathrm{i} z h_{a}} V\left(a, e^{2 \pi \mathrm{i} z}-1\right)=\sum_{n} a_{[n]} z^{-n-h_{a}} .
$$

The different modes are related to one another via

$$
a_{[m]}=(2 \pi \mathrm{i})^{-m-h_{a}} \sum_{j \geq m} c\left(h_{a}, j+h-1, m+h-1\right) a_{j},
$$

where

$$
(\log (1+z))^{m}(1+z)^{h_{a}-1}=\sum_{j \geq m} c\left(h_{a}, j, m\right) z^{j} .
$$


This defines a new vertex operator algebra with an energy tensor whose modes $L_{[n]}$ are given by

$$
L_{[n]}=(2 \pi \mathrm{i})^{-n} \sum_{j \geq n+1} c(2, j, n+1) L_{j-1}-(2 \pi \mathrm{i})^{2} \frac{c}{24} \delta_{n,-2}
$$

The second term on the right-hand side comes from the fact that $L$ is only quasiprimary, and thus picks up an anomaly under the coordinate transformation (A.3). Note that compared to (A.4) we have chosen a slightly different overall normalization so as to obtain the standard commutation relations for the modes $L_{[n]}$. To maintain the standard $N=2$ commutation relations, we also choose an analogous normalization for the $J_{[n]}$ and $G_{[n]}^{ \pm}$, i.e., without the prefactor $(2 \pi \mathrm{i})^{-h_{a}}$. With these conventions their commutation relations are

$$
\begin{aligned}
{\left[L_{[m]}, L_{[n]}\right] } & =(m-n) L_{[m+n]}+\frac{\tilde{c}}{4} m\left(m^{2}-1\right) \delta_{m,-n}, \\
{\left[L_{[m]}, J_{[n]}\right] } & =-n J_{[m+n]}, \\
{\left[L_{[m]}, G_{[n]}^{ \pm}\right] } & =\left(\frac{1}{2} m-n\right) G_{[m+n]}^{ \pm}, \\
{\left[J_{[m]}, J_{[n]}\right] } & =\tilde{c} m \delta_{m,-n}, \\
{\left[J_{[m]}, G_{[n]}^{ \pm}\right] } & = \pm G_{[m+n]}^{ \pm}, \\
\left\{G_{[m]}^{+}, G_{[n]}^{-}\right\} & =2 L_{[m+n]}+(m-n) J_{[m+n]}+\tilde{c}\left(m^{2}-\frac{1}{4}\right) \delta_{m,-n}, \\
\left\{G_{[m]}^{+}, G_{[n]}^{+}\right\} & =\left\{G_{[m]}^{-}, G_{[n]}^{-}\right\}=0,
\end{aligned}
$$

as well as

$$
o\left(J_{[-1]} \Omega\right)=(2 \pi \mathrm{i}) J_{0}
$$

\section{A.2. Recursion relations}

Zhu's original argument [2] was generalized to superalgebras in [13]. We will adapt it in the following $N=2$ case to extract the recursion relation (2.4) we shall need. 
We define the elliptic genus amplitude by

$$
\begin{aligned}
& G_{\mathcal{R}}\left(\left(a^{1}, z_{1}\right), \ldots,\left(a^{m}, z_{m}\right) ; q, y\right) \\
& \quad=\prod_{i=1}^{m} z_{i}^{h_{i}} \operatorname{Tr}_{\mathcal{R}}\left(V\left(a^{1}, z_{1}\right) \cdots V\left(a^{m}, z_{m}\right) q^{L_{0}} y^{J_{0}}(-1)^{F}\right),
\end{aligned}
$$

where $h_{i}$ is the conformal weight of the state $a^{i}$ (with respect to $L_{0}$ ) and $\mathcal{R}$ is the (irreducible) Ramond sector representation in which the trace is being taken.

There are two key identities from which the recursion relations can be deduced. The first identity is

$$
\begin{aligned}
G_{\mathcal{R}} & \left((a, w),\left(a^{1}, z_{1}\right),\left(a^{2}, z_{2}\right), \ldots,\left(a^{n}, z_{n}\right) ; q, y\right) \\
(\mathrm{A} .9)= & \sum_{j=1}^{n}\left[ \pm \sum_{m \in \mathbb{N}_{0}} \hat{\mathcal{P}}_{m+1}\left(\frac{z_{j}}{w}, q, y^{Q}\right)\right. \\
& \left.\times G_{\mathcal{R}}\left(\left(a^{1}, z_{1}\right),\left(a^{2}, z_{2}\right), \ldots,\left(a_{\left[m-h_{a}+1\right]} a^{j}, z_{j}\right), \ldots,\left(a^{n}, z_{n}\right) ; q, y\right)\right] .
\end{aligned}
$$

Here $a$ is a state with non-vanishing $U(1)$-charge, $J_{0} a=Q a \neq 0$, and the function $\hat{\mathcal{P}}_{k}\left(q_{w}, q, y\right)$ is defined in Appendix C. It is meromorphic in $q_{w}$ and has a pole at $q_{w}=1$. The \pm sign in (A.9) depends in the obvious manner on whether $a$ and the other fields $a^{j}$ are fermionic or bosonic; in particular, if $a$ is bosonic, there is no minus sign. It should also be clear that (up to the obvious \pm signs) the elliptic genus is independent of the order in which the fields appear.

The proof of (A.9) is a straightforward extension of the original argument due to Zhu [2] (see also the proof of Theorem 3.6 in [13]). The basic idea is to expand the vertex operator $(a, w)$ in terms of modes as in (A.1). Each mode is then commuted (or anti-commuted) through the trace until it returns to its place; since it picks up a non-trivial factor upon going through $y^{J_{0}}$ and $q^{L_{0}}$, we can solve for it in terms of the commutators and anti-commutators; this leads to the recursion relation (A.9). 
The second key identity is

$$
\begin{aligned}
G_{\mathcal{R}} & \left(\left(a_{\left[-h_{a}\right]} a^{1}, z_{1}\right),\left(a^{2}, z_{2}\right), \ldots,\left(a^{n}, z_{n}\right) ; q, y\right) \\
& =\sum_{k=1}^{\infty} \hat{G}_{k}\left(q, y^{Q}\right) G_{\mathcal{R}}\left(\left(a_{\left[k-h_{a}\right]} a^{1}, z_{1}\right),\left(a^{2}, z_{2}\right), \ldots,\left(a^{n}, z_{n}\right) ; q, y\right)
\end{aligned}
$$

$$
\begin{aligned}
& +\sum_{j=2}^{n} \pm \sum_{m \in \mathbb{N}_{0}} \hat{\mathcal{P}}_{m+1}\left(\frac{z_{j}}{z_{1}}, q, y^{Q}\right) \\
& \times G_{\mathcal{R}}\left(\left(a^{1}, z_{1}\right),\left(a^{2}, z_{2}\right), \ldots,\left(a_{\left[m-h_{a}+1\right]} a^{j}, z_{j}\right), \ldots,\left(a^{n}, z_{n}\right) ; q, y\right),
\end{aligned}
$$

where $\hat{G}_{k}(q, y)$ is the generalized Eisenstein series defined in Appendix C.

Since the proof in [13] is rather sketchy, we will spell it out in more detail. First note that $a_{\left[-h_{a}\right]} a^{1}$ is not homogeneous with respect to $L_{0}$. In order to insert it, nevertheless, into the elliptic genus, we extend the definition of (A.8) by linearity. We expand the first argument of $G_{\mathcal{R}}$ in (A.10) to get

$$
\begin{aligned}
\left(a_{\left[-h_{a}\right]} a^{1}, z_{1}\right) & =\sum_{i \geq-1} c_{i}\left(a_{i-h_{a}+1} a^{1}, z_{1}\right) \\
& =\sum_{i \geq-1} c_{i} \operatorname{Res}_{w-z_{1}}\left(w-z_{1}\right)^{i} V\left(V\left(a, w-z_{1}\right) a^{1}, z_{1}\right) \\
& =\sum_{i \geq-1} c_{i} \operatorname{Res}_{w-z_{1}}\left(w-z_{1}\right)^{i} V(a, w) V\left(a^{1}, z_{1}\right)
\end{aligned}
$$

where we have defined $c_{i}=c\left(h_{a}, i,-1\right)$. Taking into account the additional factors of $z_{1}$ and $w$ that come from the definition (A.8) of $G_{\mathcal{R}}$, we obtain the sum

$$
\sum_{i \geq-1} c_{i}\left(w-z_{1}\right)^{i} z_{1}^{h_{a}-1-i} w^{-h_{a}}=w^{-1}\left(\log \left(\frac{w}{z_{1}}\right)\right)^{-1}
$$

We now apply (A.9) to our expression, obtaining terms of the form

$$
\begin{gathered}
G_{\mathcal{R}}\left(\left(a^{1}, z_{1}\right), \ldots,\left(a_{\left[m-h_{a}+1\right]} a^{j}, z_{j}\right), \ldots,\left(a^{n}, z_{n}\right) ; q, y\right) \\
\times \int_{C} w^{-1}\left(\log \left(\frac{w}{z_{1}}\right)\right)^{-1} \hat{\mathcal{P}}_{m+1}\left(\frac{z_{j}}{w}, q, y^{Q}\right) d w
\end{gathered}
$$

where the contour $C$ is around $z_{1}$. 
For $j \neq 1, \hat{\mathcal{P}}_{m+1}\left(\frac{z_{j}}{w}, q, y^{Q}\right)$ is regular at $w=z_{1}$, so that we obtain directly the last two lines of (A.10). For $j=1$, however, $\hat{\mathcal{P}}_{m+1}\left(\frac{z_{1}}{w}, q, y^{Q}\right)$ has a pole at $w=z_{1}$. We perform the change of variable $w=z_{1} \exp \left(-2 \pi \mathrm{i} w^{\prime}\right)$, which yields $\operatorname{Res}_{w^{\prime}} \hat{\mathcal{P}}_{m+1}\left(\mathrm{e}^{2 \pi \mathrm{i} w^{\prime}}, q, y^{Q}\right)$. Using expansion (C.32) then gives the first term of (A.10).

The recursion relation (2.4) then follows directly from (A.10) by comparing the $z^{0}$ coefficient for $n=1$.

\section{Appendix B. Weak Jacobi forms}

A weak Jacobi form [21] of weight $k$ and index $m$ is a function $f(\tau, z)$ on $\mathbb{H}_{+} \times \mathbb{C}$ satisfying the transformation property

(B.13)

$$
f\left(\frac{a \tau+b}{c \tau+d}, \frac{z}{c \tau+d}\right)=(c \tau+d)^{k} \mathrm{e}^{\frac{2 \pi \mathrm{i} m c z^{2}}{c \tau+d}} f(\tau, z), \quad\left(\begin{array}{ll}
a & b \\
c & d
\end{array}\right) \in \operatorname{SL}(2, \mathbb{Z}),
$$

and

$$
f(\tau, z+r \tau+s)=\mathrm{e}^{-2 \pi \mathrm{i} m\left(r^{2} \tau+2 r z\right)} f(\tau, z)
$$

with $r, s \in \mathbb{Z}$. Moreover, it must have a Fourier expansion of the form

$$
f(\tau, z)=\sum_{n=0}^{\infty} \sum_{l \in \mathbb{Z}} c(n, l) q^{n} y^{l}
$$

where as usual

$$
q=\mathrm{e}^{2 \pi \mathrm{i} \tau}, \quad y=\mathrm{e}^{2 \pi \mathrm{i} z} .
$$

The space of all weak Jacobi forms is generated by the two Eisenstein series $G_{4}(q)$ and $G_{6}(q)$ (which are conventional modular forms of weight 4 and 6 , respectively) and the two weak Jacobi forms

$$
\phi_{-2,1}=\frac{\phi_{10,1}}{\Delta}, \quad \phi_{0,1}=\frac{\phi_{12,1}}{\Delta},
$$

where $\Delta=q \prod_{n=1}^{\infty}\left(1-q^{n}\right)^{24}$ and

$$
\phi_{10,1}=\frac{1}{576 \zeta(4) \zeta(6)}\left(G_{6} G_{4,1}-G_{4} G_{6,1}\right), \quad \phi_{12,1}=\frac{1}{576}\left(\frac{G_{4}^{2} G_{4,1}}{2 \zeta(4)^{3}}-\frac{G_{6} G_{6,1}}{\zeta(6)^{2}}\right) .
$$

Here $\zeta(2 k)$ is the Riemann zeta function and $G_{4,1}$ and $G_{6,1}$ are Jacobi forms defined in [21]. On general grounds, one can show that the elliptic genus of 
an $N=2$ conformal field theory of central charge $c$ is a weak Jacobi form of weight 0 and index $m=\frac{c}{6}$.

\section{Appendix C. Twisted Eisenstein series}

\section{C.1. Ordinary Eisenstein series}

The ordinary Eisenstein series are defined by

$$
\begin{aligned}
G_{2 k}(\tau) & =\sum_{(m, n) \neq(0,0)} \frac{1}{(m \tau+n)^{2 k}} \quad k \geq 2, \\
G_{2}(\tau) & =\frac{\pi^{2}}{3}+\sum_{m \in \mathbb{Z}-\{0\}} \sum_{n \in \mathbb{Z}} \frac{1}{(m \tau+n)^{2}} .
\end{aligned}
$$

They can also be written as

$$
G_{2 k}(\tau)=2 \zeta(2 k)+\frac{2(2 \pi \mathrm{i})^{2 k}}{(2 k-1) !} \sum_{n=1}^{\infty} \frac{n^{2 k-1} q^{n}}{1-q^{n}} .
$$

For $k \geq 2, G_{2 k}(\tau)$ is a modular form of weight $2 k$, i.e.,

$$
G_{2 k}\left(\frac{a \tau+b}{c \tau+d}\right)=(c \tau+d)^{2 k} G_{2 k}(\tau),
$$

whereas $G_{2}(\tau)$ transforms as

$$
G_{2}\left(\frac{a \tau+b}{c \tau+d}\right)=(c \tau+d)^{2} G_{2}(\tau)-2 \pi \mathrm{i} c(c \tau+d) .
$$

\section{C.2. Twisted Eisenstein series}

For $|q|<\left|q_{w}\right|<1$ and $y \neq 1$ define

$$
\begin{aligned}
\hat{\mathcal{P}}_{m}\left(q_{w}, q, y\right):= & \frac{(2 \pi \mathrm{i})^{m}}{(m-1) !}\left(\sum_{n=1}^{\infty} \frac{n^{m-1} q_{w}^{n}}{1-q^{n} y^{-1}}+\frac{(-1)^{m} n^{m-1} q_{w}^{-n} q^{n} y}{1-q^{n} y}\right) \\
& +\delta_{1, m} \frac{2 \pi \mathrm{i}}{1-y^{-1}}
\end{aligned}
$$


Note that $\hat{\mathcal{P}}_{m}$ is a special case of the twisted Weierstrass function $P_{m}$ introduced in $[13,14]$. More precisely,

$$
\hat{\mathcal{P}}_{m}\left(q_{w}, q, y\right)=(-2 \pi \mathrm{i})^{m} P_{m}\left[\begin{array}{c}
y^{-1} \\
1
\end{array}\right](w, \tau) .
$$

$\hat{\mathcal{P}}_{m}\left(q_{w}, q, y\right)$ converges for $|q|<\left|q_{w}\right|<1$, and

$$
\frac{\partial}{\partial w} \hat{\mathcal{P}}_{m}(w, q, y)=2 \pi \mathrm{i} q_{w} \frac{d}{d q_{w}} \hat{\mathcal{P}}_{m}(w, q, y)=m \hat{\mathcal{P}}_{m+1}(w, q, y)
$$

$\hat{\mathcal{P}}_{1}\left(q_{w}, q, y\right)$ has a simple pole at $q_{w}=1$, as can be seen by rewriting (C.27)

$$
\hat{\mathcal{P}}_{1}\left(q_{w}, q, y\right)=\frac{2 \pi \mathrm{i}}{1-q_{w}}-2 \pi \mathrm{i}+\frac{2 \pi \mathrm{i}}{1-y^{-1}}+2 \pi \mathrm{i} \sum_{n=1}^{\infty}\left(\frac{q_{w}^{n} q^{n} y^{-1}}{1-q^{n} y^{-1}}-\frac{q_{w}^{-n} q^{n} y}{1-q^{n} y}\right)
$$

where the sum is seen to be convergent for $|q|<\left|q_{w}\right|<|q|^{-1}$. Choose $q_{w}$ so that $1<\left|q_{w}\right|<|q|^{-1}$. The series then converges for both $q_{w}$ and $q q_{w}$, and a straightforward calculation shows the identity

$$
\hat{\mathcal{P}}_{1}\left(q q_{w}, q, y\right)=y \hat{\mathcal{P}}_{1}\left(q_{w}, q, y\right)
$$

Writing $q_{w} \equiv \mathrm{e}^{2 \pi \mathrm{i} w}$, we expand (C.27) in $w$. The last term gives

$$
2 \pi \mathrm{i} \sum_{n=1}^{\infty} \frac{1}{k !}(2 \pi \mathrm{i})^{k}\left(\frac{n^{k} q^{n} y^{-1}}{1-q^{n} y^{-1}}-\frac{(-n)^{k} q^{n} y}{1-q^{n} y}\right) \times w^{k}
$$

whereas the first term is

$$
-\frac{1}{w} \sum_{n=0}^{\infty} \frac{B_{n}}{n !}(2 \pi \mathrm{i} w)^{n}=-\frac{1}{w}+\pi \mathrm{i}+\sum_{n=1}^{\infty} 2 \zeta(2 n) \times w^{2 n-1} .
$$

Here, we have used

$$
\frac{x}{\mathrm{e}^{x}-1}=\sum_{n=0}^{\infty} \frac{B_{n}}{n !} x^{n}
$$

where the Bernoulli numbers $B_{n}$ are given by $B_{0}=1, B_{1}=-\frac{1}{2}$, and for $n \geq 1, B_{2 n+1}=0$ and $B_{2 n}=(-1)^{n-1} 2(2 n) !(2 \pi)^{-2 n} \zeta(2 n)$. In total, we thus 
obtain

$$
\hat{\mathcal{P}}_{1}(w, q, y)=-\frac{1}{w}+\hat{G}_{1}(q, y)+\sum_{k=2}^{\infty} \hat{G}_{k}(q, y) w^{k-1}
$$

where we have introduced the twisted Eisenstein series $\hat{G}_{k}(q, y)$ defined in (2.6). We can then use identity (C.26) to extract the poles of $\hat{\mathcal{P}}_{m}$.

\section{C.3. Transformation properties of the twisted Eisenstein series}

In order to determine the modular behaviour of $\hat{G}_{k}$, it is sufficient to consider the actions $\tau \mapsto \tau+1, z \mapsto z$ and $\tau \mapsto-1 / \tau, z \mapsto z / \tau$. $\hat{G}_{k}$ is obviously invariant under the first, while under the second we claim that the transformation is

$$
(2 \pi \mathrm{i})^{-m} \hat{G}_{m}\left(-\frac{1}{\tau}, \frac{z}{\tau}\right)=\sum_{k=1}^{m} \frac{(-1)^{m-k}}{(m-k) !}(2 \pi \mathrm{i})^{-k} \hat{G}_{k}(\tau, z) z^{m-k} \tau^{k}-(-1)^{m} \frac{z^{m}}{m !} .
$$

Note that this is very similar to the transformation properties of $G_{2}(\tau)$ : it transforms almost as a form of weight $m$ and index 0 , but has additional anomalous terms. To prove (C.33), let us assume for the moment that $\hat{G}_{1}$ transforms as

$$
\hat{G}_{1}\left(-\frac{1}{\tau}, \frac{z}{\tau}\right)=\tau \hat{G}_{1}(\tau, z)+(2 \pi \mathrm{i}) z
$$

We can then prove (C.33) by recurrence using

$$
\frac{\partial}{\partial z} \hat{G}_{m+1}(\tau, z)=-\frac{1}{m} 2 \pi \mathrm{i} \frac{\partial}{\partial \tau} \hat{G}_{m}(\tau, z) .
$$

Introducing variables $\tilde{\tau}=-1 / \tau, \tilde{z}=z / \tau$ and using

$$
\partial_{\tilde{\tau}}=\tau^{2} \partial_{\tau}+\tau z \partial_{z}, \quad \partial_{\tilde{z}}=\tau \partial_{z}
$$

we obtain

$$
\partial_{z} \hat{G}_{m+1}\left(-\frac{1}{\tau}, \frac{z}{\tau}\right)=-\frac{1}{m} 2 \pi \mathrm{i}\left(\tau \partial_{\tau}+z \partial_{z}\right) \hat{G}_{m}\left(-\frac{1}{\tau}, \frac{z}{\tau}\right) .
$$

A straightforward calculation shows that this is equal to $\partial_{z}$ of the righthand side of (C.33) for $m+1$. We have thus shown that (C.33) holds up 
to some function $f(\tau)$. To see that $f=0$, consider the limit $z \rightarrow 0$. Since $\hat{G}_{2 k}(\tau, 0)=G_{2 k}(\tau)$, the known transformation properties of the ordinary Eisenstein series fix $f=0$ for the even case. In the odd case the same is true since $\hat{G}_{2 k+1}(\tau, 0)=0$. The only somewhat subtle case occurs for $\hat{G}_{2}$. In this case $\lim _{z \rightarrow 0} z \hat{G}_{1}(\tau, z)=1$ produces the desired modular anomaly so that again $f=0$.

Let us briefly sketch how to prove (C.34). One can show that $\hat{G}_{1}$ can be rewritten as

$$
\hat{G}_{1}(\tau, z)=-\sum_{n>0} \sum_{k>0} S_{n k}-\sum_{n>0} \frac{2 z}{(n \tau)^{2}-z^{2}}-\sum_{k>0} \frac{2 z}{k^{2}-z^{2}}+\frac{1}{z}
$$

where

$$
S_{n k}=\frac{2 z}{(k+n \tau)^{2}-z^{2}}+\frac{2 z}{(-k+n \tau)^{2}-z^{2}} .
$$

Taking $\tau \mapsto-1 / \tau, z \mapsto z / \tau$, the last three terms of (C.38) transform to $\tau$ times themselves. The first sum transforms as

$$
\sum_{n>0} \sum_{k>0} S_{n k} \mapsto \tau \sum_{n>0} \sum_{k>0} S_{k n}=\tau \sum_{k>0} \sum_{n>0} S_{n k},
$$

which is formally $\tau$ times the original expression. Since the series is not absolutely convergent however, the order of summation matters. By using standard methods (see, e.g., [22]), one can show that exchanging the summation order produces exactly the modular anomaly of (C.34).

Another possibility is to derive (C.33) directly from the transformation properties of the twisted Weierstrass functions [13].

\section{References}

[1] E. P. Verlinde, Fusion rules and modular transformations in 2D conformal field theory, Nucl. Phys. B 300 (1988), 360.

[2] Y. Zhu, Vertex operator algebras, elliptic functions and modular forms, Caltech preprint (1990), J. Am. Math. Soc. 9 (1996), 237.

[3] S. D. Mathur, S. Mukhi and A. Sen, On the classification of rational conformal field theories, Phys. Lett. B 213 (1988), 303.

[4] S. D. Mathur, S. Mukhi and A. Sen, Reconstruction of conformal field theories from modular geometry on the torus, Nucl. Phys. B 318 (1989), 483. 
[5] T. Eguchi and H. Ooguri, Conformal and current algebras on general Riemann surface, Nucl. Phys. B 282 (1987), 308.

[6] G. Anderson and G. W. Moore, Rationality in conformal field theory, Commun. Math. Phys. 117 (1988), 441.

[7] M. R. Gaberdiel, Constraints on extremal self-dual CFTs, JHEP 0711 (2007), 087, arXiv:0707.4073 [hep-th].

[8] M. R. Gaberdiel and C. A. Keller, Modular differential equations and null vectors, JHEP 0809 (2008), 079, arXiv:0804.0489 [hep-th].

[9] T. Kawai, Y. Yamada and S. K. Yang, Elliptic genera and $N=2$ superconformal field theory, Nucl. Phys. B 414 (1994), 191, arXiv:hep-th/9306096.

[10] C. Eicher, Jacobi invariance of elliptic genera of $N=2$ superconformal vertex algebras, Master thesis, ETH Zürich (2009), to appear.

[11] A. Libgober, Elliptic genera, real algebraic varieties and quasi-Jacobi forms, arXiv:0904.1026 [math.AG].

[12] Y. Choie and W. Eholzer, Rankin-Cohen operators for Jacobi and Siegel forms, J. Number Theory 68 (1998), 160, arXiv:alg-geom/9611032.

[13] G. Mason, M. P. Tuite and A. Zuevsky, Torus $N$-point functions for $R$ graded vertex operator superalgebras and continuous fermion orbifolds, Commun. Math. Phys. 283 (2008), 305, arXiv:0708.0640 [math.QA].

[14] C. Dong, H. Li and G. Mason, Modular invariance of trace functions in orbifold theory, Commun. Math. Phys. 214 (2000), 1, arXiv:q-alg/9703016.

[15] G. Höhn, Selbstduale Vertexoperatorsuperalgebren und das Babymonster, Ph.D. thesis (Bonn 1995), Bonner Mathematische Schriften 286 (1996), 1, arXiv:0706.0236 [math.QA].

[16] E. Witten, Three-dimensional gravity revisited, arXiv:0706.3359 [hep-th].

[17] S. Hellerman, A universal inequality for CFT and quantum gravity, arXiv:0902.2790 [hep-th].

[18] M. R. Gaberdiel, S. Gukov, C. A. Keller, G. W. Moore and H. Ooguri, Extremal $N=(2,2)$ 2D conformal field theories and constraints of modularity, Commun. Number Theory Phys. 2 (2008), 743, arXiv:0805.4216 [hep-th]. 
[19] E. Witten, On the Landau-Ginzburg description of $N=2$ minimal models, Int. J. Mod. Phys. A 9 (1994), 4783, arXiv:hep-th/9304026.

[20] W. Eholzer and M. R. Gaberdiel, Unitarity of rational $N=2$ superconformal theories, Commun. Math. Phys. 186 (1997), 61, arXiv:hep-th/9601163.

[21] M. Eichler and D. Zagier, The theory of Jacobi forms, Birkhäuser, 1985.

[22] N. Koblitz, Introduction to elliptic curves and modular forms, SpringerVerlag, Berlin, 1984.

Institut für Theoretische Physik

ETH ZÜRICH

ZÜRICH 8093

SWITZERLAND

E-mail address: gaberdiel@itp.phys.ethz.ch

JEFFERSON PHySICAL LABORATORY

HARVARD UNIVERSITY

Cambridge, MA 02138

USA

E-mail address: ckeller@physics.harvard.edu

RECEIVED APRIL 21, 2009 